Mizzi, S. and Emerson, D.R. and Stefanov, S. and Barber, R.W. and Reese, J.M. (2007) Effects of rarefaction on cavity flow in the slip regime. Journal of Computational and Theoretical Nanoscience, 4 (4). pp. 817-822. ISSN 1546-1955

http://eprints.cdlr.strath.ac.uk/6661/

Strathprints is designed to allow users to access the research output of the University of Strathclyde. Copyright (C) and Moral Rights for the papers on this site are retained by the individual authors and/or other copyright owners. You may not engage in further distribution of the material for any profitmaking activities or any commercial gain. You may freely distribute both the url (http://eprints.cdlr.strath.ac.uk) and the content of this paper for research or study, educational, or not-for-profit purposes without prior permission or charge. You may freely distribute the url (http://eprints.cdlr.strath.ac.uk) of the Strathprints website.

Any correspondence concerning this service should be sent to The Strathprints Administrator: eprints@cis.strath.ac.uk 


\title{
Effects of Rarefaction on Cavity Flow in the Slip Regime
}

\author{
Simon Mizzi ${ }^{1,2, *}$, David R. Emerson², Stefan K. Stefanov ${ }^{3}$, \\ Robert W. Barber ${ }^{2}$, and Jason M. Reese ${ }^{1}$ \\ ${ }^{1}$ Department of Mechanical Engineering, University of Strathclyde, Glasgow G1 1XJ, United Kingdom \\ ${ }^{2}$ Centre for Microfluidics and Microsystems Modelling, CCLRC Daresbury Laboratory, \\ Warrington WA4 4AD, United Kingdom \\ ${ }^{3}$ Institute of Mechanics, Bulgarian Academy of Sciences, Acad. G. Bonchev Str., BI. 4, Sofia 1113, Bulgaria
}

\begin{abstract}
The Navier-Stokes-Fourier equations, with boundary conditions that account for the effects of velocity-slip and temperature-jump, are compared to the direct simulation Monte Carlo method for the case of a lid-driven micro-cavity. Results are presented for Knudsen numbers within the slipflow regime where the onset of nonequilibrium effects are usually observed. Good agreement is found in predicting the general features of the velocity field and the recirculating flow. However, although the steady-state pressure distributions along the walls of the driven cavity are generally in good agreement with the Monte Carlo data, there is some indication that the results are starting to show noticeable differences, particularly at the separation and reattachment points. The modified Navier-Stokes-Fourier equations consistently overpredict the maximum and minimum pressure values throughout the slip regime. This highlights the need for alternative boundary formulations or modeling techniques that can provide accurate and computationally economic solutions over a wider range of Knudsen numbers.
\end{abstract}

Keywords: Microfluidics, Cavity Flow, Slip Regime, Non-Equilibrium Phenomena, Knudsen Number.

\section{INTRODUCTION}

The use of micro-electro-mechanical systems (MEMS) has been proposed in many applications, including industrial engineering, biomedical analyses, environmental control, micro-processor cooling and high-precision printing. As a result, terms such as micro-ducts, micro-heat-exchangers, micro-pumps, and micro-sensors are now commonly used in many diverse fields. One area where the research community is particularly active is trying to understand gas dynamics in micron and sub-micron sized domains. The flow characteristics in miniaturized systems are known to differ significantly from those found in conventional devices. For example, the Navier-Stokes-Fourier (NSF) equations with no-slip boundary conditions are no longer valid when the characteristic length scale enters the micron range. ${ }^{1}$

The inadequacy of the NSF equations in modeling gas dynamics in micron-sized domains can be explained by the fact that they are only able to describe flows that are close to thermodynamic equilibrium. However, at small length scales, nonequilibrium effects are frequently observed in

\footnotetext{
${ }^{*}$ Author to whom correspondence should be addressed.
}

gas flows. Collisions between the molecules are the only mechanism for a gas to maintain equilibrium. If a gas is too rarefied or confined in a micro-geometry, the number of intermolecular collisions will be significantly reduced and nonequilibrium effects will start to dominate. The degree of rarefaction of a gas is defined through the Knudsen number, $\mathrm{Kn}$, which is given by $\mathrm{Kn}=\lambda / L$, where $\lambda$ is the mean free path (i.e. the average distance travelled by the gas molecules between successive collisions) and $L$ is the characteristic size of the domain.

The majority of numerical studies to date have mainly involved simple geometries, such as planar channels (Couette flow, Poiseuille flow) or annular configurations (cylindrical Couette flow), where the aim has been to improve our understanding of near-wall flow phenomena. In this paper, we focus on how the nonequilibrium physics will affect the predicted flow field in a geometrically simple but complex flow environment. The lid-driven cavity will therefore be used to highlight how the NSF equations, modified to take into account the effects of velocity-slip and temperature-jump, perform in a recirculating flow. In the absence of experimental data, the NSF predictions are compared to results obtained using the direct simulation Monte Carlo (DSMC) method. 


\section{MODELING APPROACHES}

The Boltzmann equation is the fundamental governing equation for a dilute gas undergoing binary collisions. The basic form of the Boltzmann equation can be written as

$$
\frac{\partial f}{\partial t}+c_{i} \frac{\partial f}{\partial x_{i}}+a_{i} \frac{\partial f}{\partial c_{i}}=\left.\frac{\partial f}{\partial t}\right|_{C}
$$

where $f$ is the particle distribution function which is a function of time, $t$, the position vector, $x_{i}$, and the molecular velocity vector, $c_{i}$. The term on the right-hand side of Eq. (1), $\partial f /\left.\partial t\right|_{C}$, is a production term for $f$ resulting from the binary collisions and is commonly referred to as the collision operator. The Boltzmann equation is able to describe gases that are in equilibrium and nonequilibrium alike but its solution is a non-trivial task due to the complexity of the collision term.

Various methods have been proposed to simplify Eq. (1) with each method attempting to retain an acceptable level of accuracy in describing the fundamental physics. There are essentially two main approaches for simulating rarefied gases; in one approach, discrete molecular modeling is used to describe the fluid through a microscopic formalism, i.e. as a collection of moving molecules which interact through collisions or very close proximity potentials. Discrete modeling can be performed using either statistical ensemble averages, as in the direct simulation Monte Carlo approach, ${ }^{2}$ or through deterministic methods, such as molecular dynamics. ${ }^{3}$ Although discrete methods achieve a realistic representation of the microscopic behavior, their application has been restricted to geometrically simple flows due to their computationally intensive nature. ${ }^{4}$ However, the information preservation (IP) method $^{5,6}$ may offer a promising approach for reducing the computational requirements of DSMC techniques while Baker and Hadjiconstantinou ${ }^{7}$ have recently demonstrated that the statistical scatter associated with Monte Carlo methods can be reduced by considering only the deviation from the equilibrium condition.

An alternative approach is to retain a continuum formulation to develop simpler representations of the Boltzmann equation. In this case, the fluid is assumed to be continuous and infinitely divisible so that velocity, density, pressure, and other properties can readily be defined at any point in space and time. One such approach is through the use of an extended hydrodynamic approximation of Eq. (1). This can be obtained by performing a ChapmanEnskog expansion, ${ }^{8}$ where the distribution function, $f$, is expanded in a power series based on the Knudsen number. The power series can be truncated at any power of $\mathrm{Kn}$ and will yield the Euler, NSF, Burnett, or higher-order descriptions. Another approach is the method of moments ${ }^{9-11}$ where the distribution function is multiplied by a function that depends only on the molecular velocity. The transport equations can then be derived from a power series involving Hermite polynomials. It should be noted that there are a number of challenges with the foregoing approaches. For example, the Burnett equations have stability issues and are unable to capture Knudsen layers whilst moment methods do not provide a closed system and also require additional boundary conditions. However, there are advantages in these approaches because they are globally second-order (or higher) in Knudsen number and will naturally recover the NSF equations when the Knudsen number is small.

Alternatively, it is possible to combine the NSF equations with simple phenomenological extensions. Such techniques include the application of velocity-slip ${ }^{12}$ and temperature-jump ${ }^{13}$ boundary conditions. It is also possible to develop second-order boundary conditions for the velocity-slip $^{14,15}$ or to derive more accurate boundary conditions based on higher-order constitutive relations, such as the Burnett equations. ${ }^{16}$ These techniques improve the accuracy of mass flow rate predictions but often fail to capture nonlinear phenomena in the near-wall region. More recently, the development of constitutive law re-scaling, in the form of a wall function, ${ }^{17}$ has been shown to offer the potential of replicating the nonlinear stress/strain behavior in the vicinity of solid walls. For the present analysis, we use a boundary formulation derived from Grad's 13 moment equations. ${ }^{11}$

\section{CLASSIFICATION OF THE FLOW REGIME}

Several distinct regimes can be defined that characterize the state of a particular flow: ${ }^{1}$

- For $\mathrm{Kn}<0.001$, the flow is in the continuum regime and the conventional no-slip boundary condition is considered to be valid since the flow is in thermodynamic equilibrium. - For $0.001<\mathrm{Kn}<0.1$, the gas is in the slip-flow regime. The NSF equations are considered to be adequate provided the effects of velocity-slip and temperature-jump at the wall are taken into account.

- For $0.1<\mathrm{Kn}<10$, the flow is said to be in the transition regime. The use of the NSF equations becomes questionable and alternative approaches are needed to model such flows using either discrete (particle-based) methods, extended hydrodynamics, or the method of moments.

- For $\mathrm{Kn}>10$, the flow is in the free-molecular regime. In this regime, the frequency of intermolecular collisions is very low and the mean free path is large compared to the characteristic length scale of the flow domain. The continuum hypothesis is no longer valid and a collisionless form of the Boltzmann equation can be used to describe the flow.

The limiting Knudsen numbers in the above classification scheme are somewhat empirical, and are generally based upon simple flows that have a predominant flow direction and pronounced gradients normal to the streamwise direction, e.g., Couette or Poiseuille flow. In the case of more complex flows, however, the boundaries between the different regimes may depend upon the particular geometric details of the problem. As will be demonstrated 
later for a driven-cavity flow, nonequilibrium effects are discernible at Knudsen numbers well below the conventionally accepted upper limit of the slip-flow regime.

\section{THE DRIVEN CAVITY PROBLEM}

Cavities, steps and cut-outs occur frequently in many engineering designs. Such configurations generate sharp changes in the flow variables and their gradients. At the macroscopic scale, modeling the flow phenomena associated with cavities is challenging, particularly at high Mach numbers. However, at the microscale, other complexities can arise due to the loss of local thermodynamic equilibrium.

The lid-driven cavity, shown schematically in Figure 1, has been extensively investigated in a completely different context since the problem is often used as a validation test for numerical schemes. Despite its geometric simplicity, the problem is rich in flow physics associated with the recirculating eddy. Many of the investigations in the literature are presented in an incompressible NSF framework and are solved using either a pressure-velocity coupling or a streamfunction-vorticity formulation. ${ }^{18,19}$ In general, the objective of these studies is to investigate the effectiveness of convective numerical schemes over a wide range of Reynolds numbers.

In the present paper, we investigate a micro-scale liddriven cavity since very few studies are available for rarefied cavity flows. Su et al. ${ }^{20}$ presented solutions obtained using the Bhatnagar-Gross-Krook (BGK) approximation of the Boltzmann equation while Jiang et al. ${ }^{21}$ compared the DSMC and information preservation (IP) methods, and investigated the validity of the IP method for low-speed flows. More recently, Naris and Valougeorgis ${ }^{22}$ have conducted a comprehensive study of the driven cavity problem over the whole Knudsen number regime using the discrete velocity method to solve the linearized Boltzmann equation. They showed that for low Mach number flows,

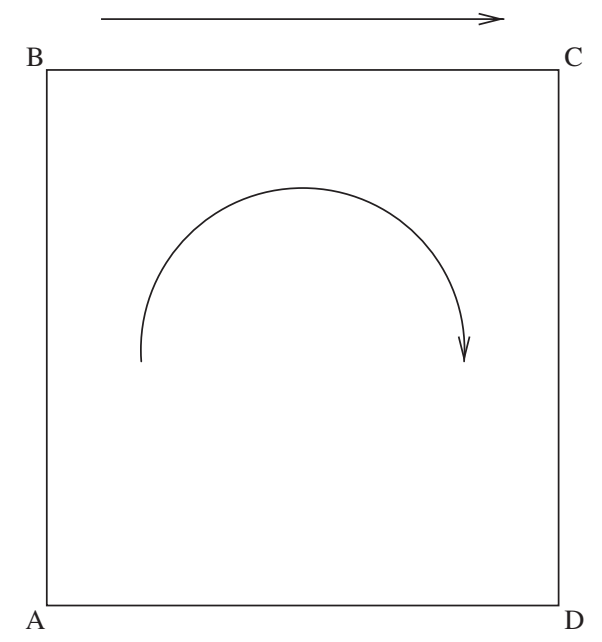

Fig. 1. Schematic diagram of a driven cavity. the temperature variations were small. In the present study, we compare NSF predictions with results obtained from the DSMC method. In particular, we highlight some of the limitations of the NSF approach in the slip-flow regime.

\section{NUMERICAL SOLUTION OF THE NSF EQUATIONS}

The Navier-Stokes-Fourier equations have been discretized on a collocated grid using the finite-volume pressurevelocity-density coupling approach proposed by Demirdzic et al. ${ }^{23}$ Since the Reynolds number is relatively small, a central-difference scheme is considered appropriate. ${ }^{24}$ The central-difference scheme was implemented at cell boundaries for both the convective and diffusive fluxes while the source terms were computed at cell centres. A meshresolution study was carried out using grids composed of $40 \times 40,80 \times 80$ and $160 \times 160$ cells. In all test cases, the results were numerically equivalent for the $80 \times 80$ and $160 \times 160$ grids. We present here only the grid-independent results.

\subsection{Velocity-Slip and Temperature-Jump Boundary Conditions}

The application of velocity-slip and temperature-jump boundary conditions in the NSF equations is a simplified phenomenological approach to represent both nonequilibrium and gas-surface interaction effects near the solid walls. These boundary conditions were first proposed by Maxwell ${ }^{12}$ and von Smoluchowski, ${ }^{13}$ respectively. Using Grad's closure approximation for the distribution function, $f$, the boundary conditions can be written as: ${ }^{11}$

$$
\begin{aligned}
& v_{i_{\text {slip }}}=v_{i_{\text {gas }}}-v_{i_{\text {wall }}} \\
& =\frac{(-(2-\sigma) / \sigma) \sqrt{\frac{\pi}{2}} \sqrt{R T}\left(\alpha_{1} \tau_{i j} n_{j}-n_{i} \tau_{j k} n_{j} n_{k}\right)-\frac{1}{5}\left(\alpha_{2} q_{i}-n_{i} q_{k} n_{k}\right)}{\rho R T+\frac{1}{2} \tau_{j k} n_{j} n_{k}}
\end{aligned}
$$

and

$\frac{T_{\text {wall }}}{T}-1$

$=\frac{\left(2-\sigma_{T} / \sigma_{T}\right) \sqrt{\frac{\pi}{2 R T}} \frac{1}{2} \beta_{1} q_{k} n_{k}+\frac{1}{4} \tau_{j k} n_{j} n_{k}}{p+\frac{1}{2} \tau_{j k} n_{j} n_{k}}-\frac{v_{i_{\text {slip }}} v_{i_{\text {slip }}}}{4 R T}$

where $v_{i_{\text {slip }}}$ is the slip velocity at the wall, $v_{i_{\text {gas }}}$ and $v_{i_{\text {wall }}}$ are the gas and wall velocities, $T_{\text {wall }}$ and $T$ are the wall and gas temperatures, $\sigma$ and $\sigma_{T}$ are the tangential momentum and energy accommodation coefficients, respectively, and $\tau_{i j}$ and $q_{i}$ are the viscous stresses and heat flux. The term, $n_{i}$, is the normal vector, $p$ is the pressure, $\rho$ is the density, and $R$ is the specific gas constant. The terms, $\alpha_{1}, \alpha_{2}$, and $\beta_{1}$ are Knudsen layer correction coefficients and are set to $\alpha_{1}=1.114, \alpha_{2}=1.34533$, and $\beta_{1}=1.127$, respectively ${ }^{11}$ while the tangential momentum and energy accommodation coefficients are assumed to be unity. A second-order one-sided difference scheme was used to determine the required gradients for the boundary conditions. 


\section{DIRECT SIMULATION MONTE CARLO SOLUTION}

The DSMC method used in this study follows the approach proposed by $\mathrm{Bird}^{2}$ where the gas molecules are represented by a much smaller number of "stochastic" particles. The algorithm is divided into two main stages consisting of translational movement of the particles and binary collisions between the particles. Solid boundaries are taken into account in the translational stage of the algorithm and a special recursive treatment is implemented in the vicinity of the corners of the cavity. A Maxwellian scattering kernel with perfect accommodation is assumed at the walls and the "no time counter" method is used to simulate the collision interactions.

In the present paper, we are interested in the steadystate solution. Since the DSMC method is a kinetic formulation (i.e. dependent on time, molecular velocity, and position), the macroscopic variables are computed using time-averaged moments over a number of kinetic time steps. The moments are spatially averaged within the cell volumes. In particular, we have computed the following moments: $m, m \bar{c}_{i}, m \overline{c_{i} c_{i}} / 2, m \overline{C_{i} C_{j}}$, and $m \overline{C_{i} C_{i} C_{j}} / 2$ where $m$ is the molecular mass and $C_{i}$ is the peculiar velocity defined as the deviation of the molecular velocity, $c_{i}$, from the average velocity $u_{i}$ (i.e. $C_{i}=c_{i}-u_{i}$ ), the overbar indicates time-averaged values and repeated indices represent the usual Einstein convention of tensor summation. These averages yield moments corresponding to the density, bulk velocity, internal energy, viscous stress, and heat flux, respectively.

The DSMC simulations employed a hard-sphere model of argon with a molecular mass of $6.63 \times 10^{-26} \mathrm{~kg}$ and a molecular diameter of $3.42 \times 10^{-10} \mathrm{~m}$. The computational domain was discretized using a uniform distribution of cells with a grid resolution of either $50 \times 50$ or $60 \times 60$ cells, depending upon the Knudsen number. Typically, the DSMC simulations employed 50 particles per cell although this was increased to approximately 300 particles per cell for the lowest Knudsen number case. Over 10 million sampling time steps were used to reduce the statistical scatter in the DSMC results.

\section{RESULTS AND DISCUSSION}

The lid-driven cavity has been investigated for two Knudsen numbers, $\mathrm{Kn}=0.05$ and $\mathrm{Kn}=0.1$. For convenience, the results are presented in a nondimensional form given by the following:

$$
\begin{aligned}
& X=\frac{x_{1}}{L}, \quad Y=\frac{x_{2}}{L}, \quad S=\frac{s}{L}, \quad P=\frac{p}{P_{o}}, \\
& U=\frac{v_{1}}{U_{\text {lid }}} \quad \text { and } \quad V=\frac{v_{2}}{U_{\text {lid }}}
\end{aligned}
$$

where $x_{1}$ and $x_{2}$ are the horizontal and vertical distances, respectively, $L$ is the cavity length, $s$ is the distance along (a)

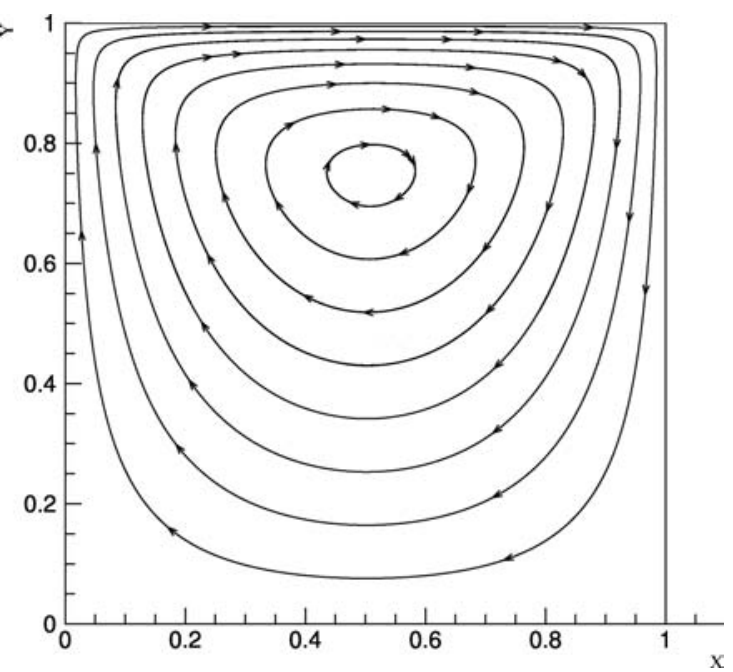

(b) $\succ$

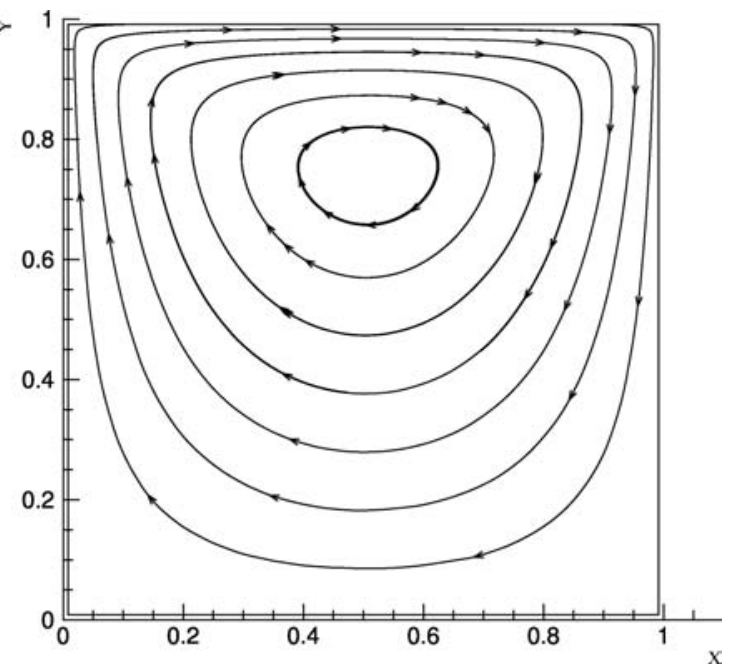

Fig. 2. Velocity streamlines for $\mathrm{Kn}=0.05$ : (a) NSF solution and (b) DSMC data.

the walls of the cavity (starting from $A$ in Fig. 1 and progressing in a clockwise direction), $P_{o}$ is the initial pressure in the cavity $\left(P_{o}=101135 \mathrm{~N} \mathrm{~m}^{-2}\right), v_{1}$ and $v_{2}$ are the velocity components in the $x_{1}$ and $x_{2}$ directions, and $U_{\text {lid }}$ is the velocity of the moving wall. The upper case symbols represent the nondimensional quantities.

Figures 2 and 3 compare the streamlines predicted by the NSF and DSMC approaches at the two Knudsen numbers. The simulations have considered a driven cavity with a unit aspect ratio. In both cases, the Mach number, defined as $M a=U_{\text {lid }} / \sqrt{2 R T_{0}}$, where $T_{0}$ is the reference temperature $(273 \mathrm{~K})$, was 0.09 . In general, the agreement between the two modeling approaches is very good and the NSF equations predict the overall features of the flow with reasonable accuracy, including the location of the centre of the eddy. Figure 4 compares the predicted velocity profiles along the centreline of the cavity $\left(x_{1} / L=0.5\right.$ and $x_{2} / L=0.5$ ). Once again, the two modeling approaches yield very similar results but the NSF equations overpredict the velocity-slip along the moving wall at $\mathrm{Kn}=0.1$. 
(a)

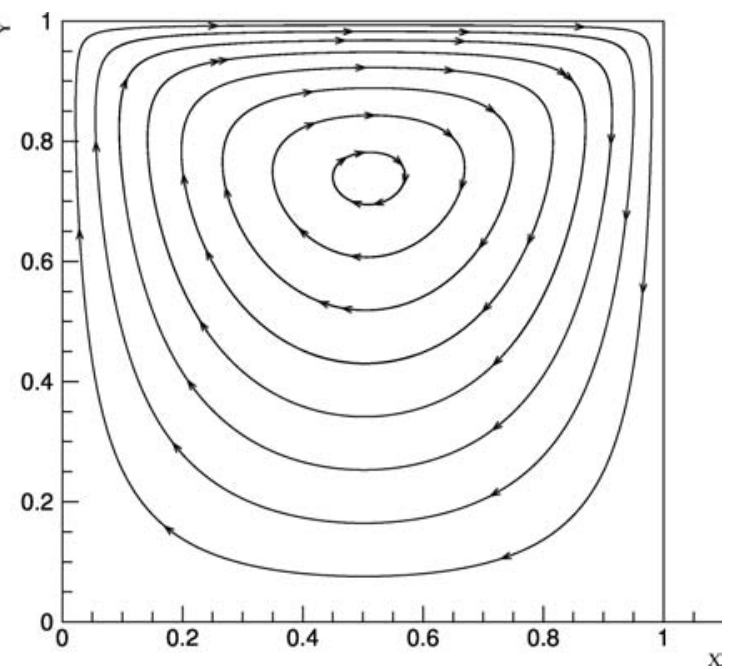

(b)

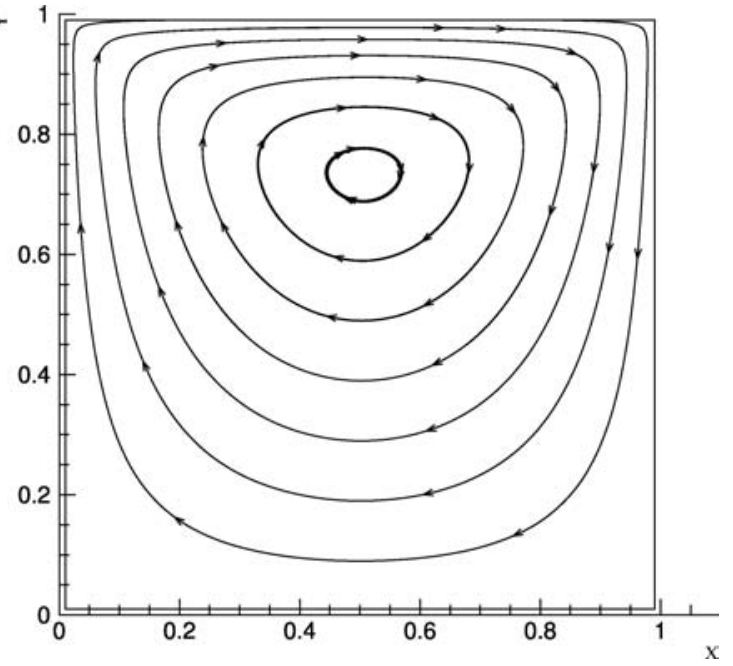

Fig. 3. Velocity streamlines for $\mathrm{Kn}=0.1$ : (a) NSF solution and (b) DSMC data.

In the cases considered, the flow field consists of a single primary recirculating eddy with the flow having insufficient inertia to generate secondary vortices in the corners of the cavity.

Figure 5 shows the nondimensional pressure distribution along the walls of the cavity. For both Knudsen numbers, it can be seen that there is reasonable agreement between the NSF solution and the DSMC predictions. However, in both cases, the NSF predictions show considerable discrepancies in the vicinity of the upper left-hand and right-hand corners of the cavity ( $S=1$ and $S=2$ ), where separation and reattachment occur. The DSMC predictions are very similiar to the pressure distributions obtained by Jiang et al. $^{21}$ In contrast, the NSF equations overestimate the pressure difference between these two corner points (B and $\mathrm{C}$ in Figure 1) leading to an incorrect pressure distribution along the moving wall of the cavity. The accurate prediction of reattachment pressures is particularly important and the present results show that, for the driven cavity problem, nonequilibrium effects are causing inaccuracies well (a)

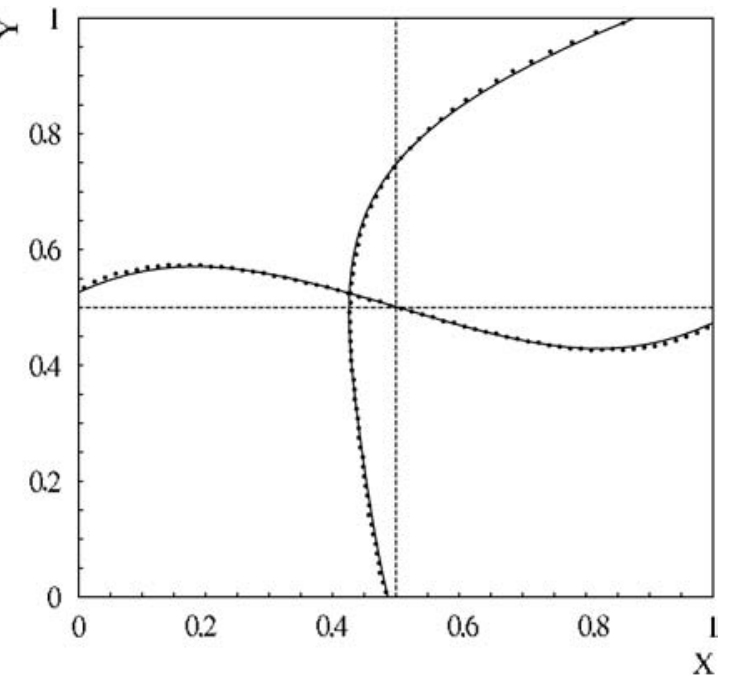

(b)

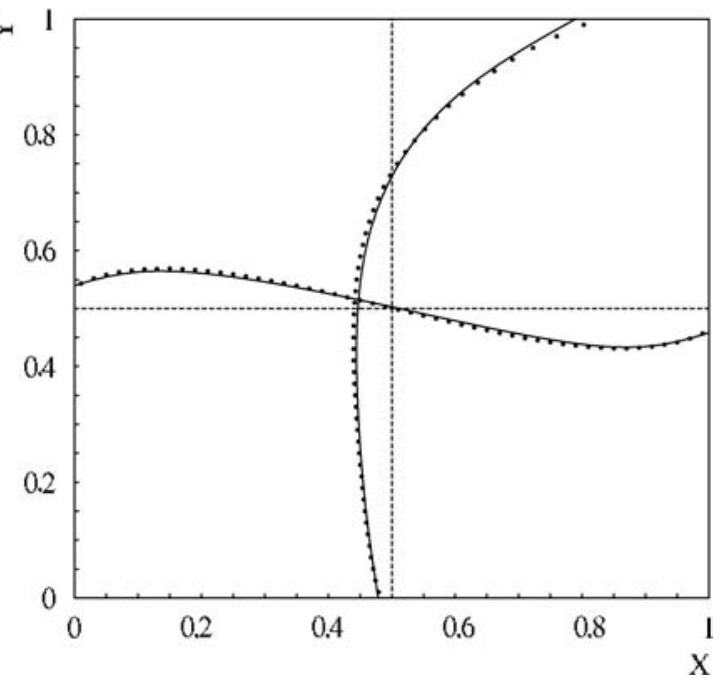

Fig. 4. Velocity profiles along the centreline of the cavity $\left(x_{1} / L=0.5\right.$ and $x_{2} / L=0.5$ ): (a) $\mathrm{Kn}=0.05$ and (b) $\mathrm{Kn}=0.1$. Comparison of DSMC data $(\bullet)$ and the NSF solution $(-)$.

before the conventionally-accepted upper limit of the slipflow regime.

\section{CONCLUSIONS}

A comparison between the Navier-Stokes-Fourier equations and the direct simulation Monte Carlo method has been carried out for a driven micro-cavity problem. The NSF equations are normally assumed to be valid up to a Knudsen number of $\mathrm{Kn}=0.1$ and good agreement has been observed for many aspects of the flow. However, for this particular problem, the predicted pressure along the moving wall is clearly affected by nonequilibrium effects resulting in an overprediction of the pressure difference at the separation and reattachment locations. Surprisingly, this feature was observed at a Knudsen number as low as 0.05 . This failure in the NSF equations was unexpected at such a low value of $\mathrm{Kn}$ and highlights the need for 
(a)

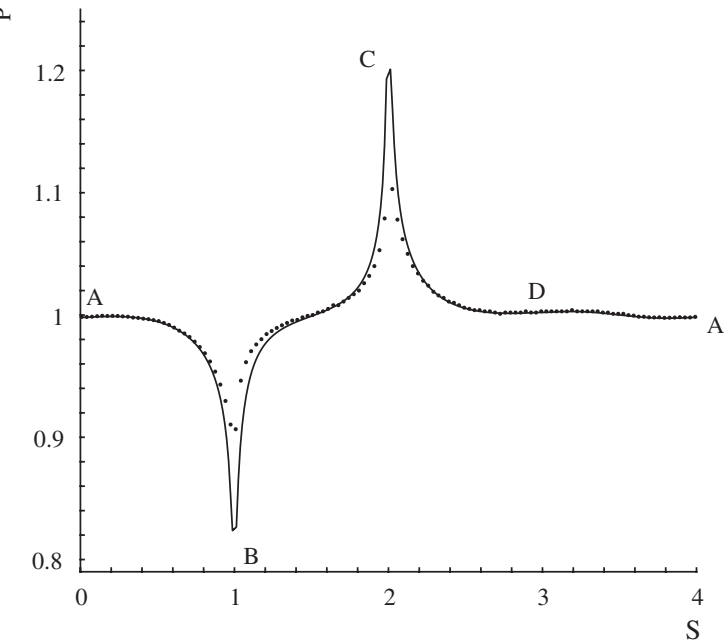

(b)

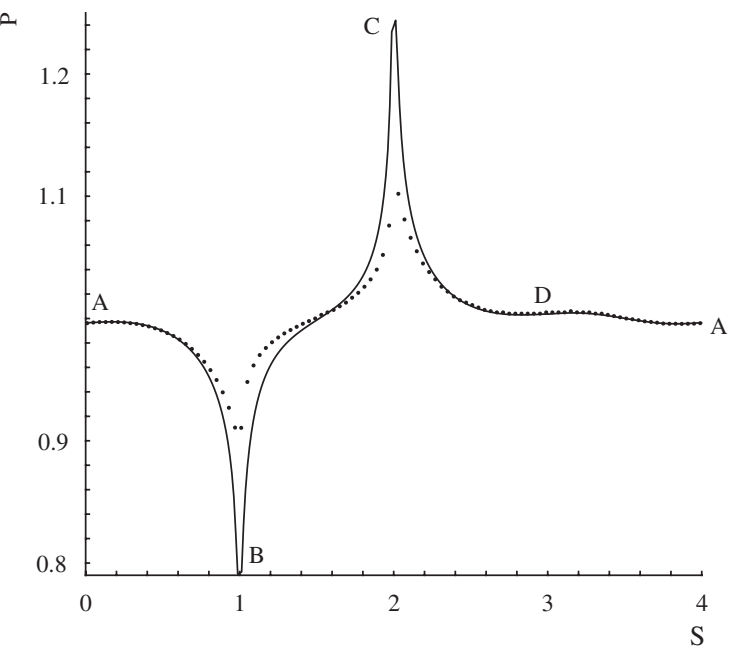

Fig. 5. Pressure distribution along the cavity walls: (a) $\mathrm{Kn}=0.05$ and (b) $\mathrm{Kn}=0.1$. Comparison of DSMC data $(\bullet)$ and the NSF solution (-).

alternative boundary treatments or modeling approaches that can provide accurate and computationally economic solutions over a wider range of Knudsen numbers.

Acknowledgments: The authors are grateful to the UK Engineering and Physical Sciences Research Council (EPSRC) for supporting this research under Grant
No. GR/S77196/01. Additional support was provided by EPSRC under the auspices of Collaborative Computational Project 12 (CCP12).

\section{References}

1. M. Gad-el-Hak, ASME J. Fluids Engrg. 121, 5 (1999).

2. G. A. Bird, Molecular Gas Dynamics and the Direct Simulation of Gas Flows, Clarendon Press, Oxford (1994).

3. D. C. Rapaport, The Art of Molecular Dynamics Simulation, Cambridge University Press, Cambridge, UK (2004).

4. E. S. Oran, C. K. Oh, and B. Z. Cybyk, Ann. Rev. Fluid Mechanics 30, 403 (1998).

5. J. Fan and C. Shen, J. Computational Phys. 167, 393 (2001).

6. Q. Sun and I. D. Boyd, Theoretical development of the information preservation method for strongly nonequilibrium flows. Proceedings of the 38th Thermophysics Conference, Toronto, Canada (2005).

7. L. L. Baker and N. G. Hadjiconstantinou, Physics of Fluids 17, 051703 (2005).

8. S. Chapman and T. G. Cowling, The Mathematical Theory of Non-Uniform Gases, Cambridge University Press, Cambridge, UK (1990).

9. H. Grad, Commun. Pure Appl. Math. 2, 331 (1949).

10. M. N. Kogan, Rarefied Gas Dynamics, Plenum Press, New York (1969).

11. H. Struchtrup, Macroscopic Transport Equations for Rarefied Gas Flows, Springer, Germany (2005).

12. J. C. Maxwell, Philos. Trans. R. Soc. London 170, 231 (1879).

13. M. von Smoluchowski, Annalen der Physik und Chemie 64, 101 (1898).

14. N. G. Hadjiconstantinou, Physics of Fluids 15, 2352 (2003).

15. R. W. Barber and D. R. Emerson, Heat Transfer Engrg. 27, 3 (2006).

16. D. A. Lockerby, J. M. Reese, D. R. Emerson, and R. W. Barber, Phys. Rev. E 70, 017303 (2004).

17. D. A. Lockerby, J. M. Reese, and M. A. Gallis, AIAA J. 43, 1391 (2005).

18. P. N. Shankar and M. D. Deshpande, Ann. Rev. Fluid Mech. 32, 93 (2000).

19. E. Erturk, T. C. Corke, and C. Gökcöl, Int. J. Numer. Meth. Fluids 48, 747 (2005).

20. M. Su, K. Xu, and M. S. Ghidaoui, J. Computational Phys. 150, 17 (1999).

21. J. Jiang, J. Fan, and C. Shen, AIP Conference Proceedings - Rarefied Gas Dynamics: 23rd International Symposium (2003), Vol. 663, p. 784.

22. S. Naris and D. Valougeorgis, Physics of Fluids 17, 097106 (2005).

23. I. Demirdzic, Z. Lilek, and M. Peric, Int. J. Numer. Meth. Fluids 16, 1029 (1993).

24. J. H. Ferziger and M. Peric, Computational Methods for Fluid Dynamics, Springer-Verlag, Germany (1996).

Received: 4 October 2006. Accepted: 23 October 2006. 